\title{
Real-world survival outcomes of heavily pretreated patients with refractory HR+, HER2-metastatic breast cancer receiving single-agent chemotherapy-a comparison with MONARCH 1
}

\author{
Hope S. Rugo ${ }^{1}$ (1) - Veronique Dieras ${ }^{2} \cdot$ Javier Cortes $^{3,4,5} \cdot$ Debra Patt $^{6,7} \cdot$ Hans Wildiers $^{8}$. Joyce O'Shaughnessy ${ }^{9}$. \\ Esther Zamora ${ }^{5}$. Denise A. Yardley ${ }^{10} \cdot$ Gebra Cuyun Carter $^{11} \cdot$ Kristin M. Sheffield $^{11} \cdot \mathrm{Li} \mathrm{Li}^{11} \cdot$ Valerie A. M. Andre ${ }^{11}$. \\ Xiaohong I. $\mathrm{Li}^{11}$ - Martin Frenzel ${ }^{11}$. Yu-Jing Huang ${ }^{11}$ - Maura N. Dickler ${ }^{11}$. Sara M. Tolaney ${ }^{12}$
}

Received: 21 May 2020 / Accepted: 25 July 2020 / Published online: 12 August 2020

(c) The Author(s) 2020

\begin{abstract}
Purpose In MONARCH 1 (NCT02102490), single-agent abemaciclib demonstrated promising efficacy activity and tolerability in a population of heavily pretreated women with refractory HR+, HER2- metastatic breast cancer (MBC). To help interpret these results and put in clinical context, we compared overall survival (OS) and duration of therapy (DoT) between MONARCH 1 and a real-world single-agent chemotherapy cohort.

Methods The real-world chemotherapy cohort was created from a Flatiron Health electronic health records-derived database based on key eligibility criteria from MONARCH 1. The chemotherapies included in the cohort were single-agent capecitabine, gemcitabine, eribulin, or vinorelbine. Results were adjusted for baseline demographics and clinical differences using Mahalanobis distance matching (primary analysis) and entropy balancing (sensitivity analysis). OS and DoT were analyzed using the Kaplan-Meier method and Cox proportional hazards regression.

Results A real-world single-agent chemotherapy cohort $(n=281)$ with eligibility criteria similar to the MONARCH 1 population $(n=132)$ was identified. The MONARCH $1(n=108)$ cohort was matched to the real-world chemotherapy cohort $(n=108)$. Median OS was 22.3 months in the abemaciclib arm versus 13.6 months in the matched real-world chemotherapy cohort with an estimated hazard ratio (HR) of 0.54 . The median DoT was 4.1 months in MONARCH 1 compared to 2.9 months in the real-world chemotherapy cohort with HR of 0.76 .

Conclusions This study demonstrates an approach to create a real-world chemotherapy cohort suitable to serve as a comparator for trial data. These exploratory results suggest a survival advantage and place the benefit of abemaciclib monotherapy in clinical context.
\end{abstract}

Keywords Abemaciclib - Electronic health records · Metastatic breast cancer · Overall survival $\cdot$ Real-world evidence . Retrospective study $\cdot$ Single-arm trial $\cdot$ Real-world control arm

Hope S. Rugo

Hope.Rugo@ucsf.edu

1 Comprehensive Cancer Center, University of California San Francisco, San Francisco, CA, USA

2 Centre Eugene Marquis UNICANCER, Rennes Cedex, France

3 IOB Institute of Oncology, Quironsalud Group, Madrid, Spain

4 IOB Institute of Oncology, Quironsalud Group, Barcelona, Spain

5 Vall D'Hebron University Hospital, Vall D'Hebron Institute of Oncology, Barcelona, Spain

\footnotetext{
6 Texas Oncology, Austin, TX, USA

7 US Oncology, Dallas, TX, USA

8 Department of General Medical Oncology, University Hospital Gasthuisberg, Leuven, Belgium

9 Texas Oncology, US Oncology, Baylor University Medical Center, Dallas, TX, USA

10 Sarah Cannon Research Institute, Tennessee Oncology PLLC, Nashville, TN, USA

11 Eli Lilly and Company, Indianapolis, IN, USA

12 Dana-Farber Cancer Institute, Boston, MA, USA
} 


\section{Introduction}

Hormone receptor positive $(\mathrm{HR}+)$, human epidermal growth factor receptor 2 negative (HER2-) is the most prevalent subtype of invasive breast cancer and accounts for approximately $70 \%$ of all cases [1]. Metastatic breast cancer (MBC) remains a universally fatal disease, with overall survival (OS) limited to 2 to 3 years on average [2]. Despite the availability of endocrine therapy for the treatment of $\mathrm{HR}+$, HER2- MBC, benefits progressively diminish with the development of resistance and progressive disease (PD).

Inhibitors of cyclin-dependent kinases (CDKs) $4 \& 6$, in combination with endocrine therapy, have been recommended by the National Comprehensive Cancer Network (NCCN) [3] and the European Society for Medical Oncology (ESMO) for treatment of HR+, HER2 - advanced breast cancer (ABC) [4]. After progression on multiple lines of endocrine-based therapy, either alone or in combination with agents such as CDK4 \& 6 inhibitors, sequential single-agent cytotoxic chemotherapy becomes the standard of care for most patients [3]. Cytotoxic chemotherapy is associated with substantially more toxicity compared to endocrine therapies [5-8]. The addition of CDK $4 \& 6$ inhibitors to endocrine therapy has markedly improved disease control in both the first and second or greater line settings and recently have been shown to improve survival in several settings [9-13].

Abemaciclib is a potent and selective oral, small-molecule inhibitor of CDK4 \& 6 which leads to sustained cell cycle arrest when dosed on a continuous schedule [14]. Abemaciclib has received regulatory approval globally in combination with an aromatase inhibitor as initial endocrine-based therapy for the treatment of postmenopausal women with $\mathrm{HR}+, \mathrm{HER} 2-\mathrm{ABC}$ or MBC, in combination with fulvestrant for the treatment of women with $\mathrm{HR}+, \mathrm{HER} 2-\mathrm{ABC}$ or $\mathrm{MBC}$ with disease progression following endocrine therapy, and in the United States (US) as a monotherapy for the treatment of adult patients with $\mathrm{HR}+, \mathrm{HER} 2-\mathrm{ABC}$ or MBC with disease progression following endocrine therapy and prior chemotherapy in the metastatic setting [3, 4, 9]. Abemaciclib has demonstrated clinical activity as monotherapy in patients with $\mathrm{HR}+$, HER2- MBC heavily pretreated with both endocrine and chemotherapy in the metastatic setting in MONARCH 1 (NCT02102490) [15].

MONARCH 1 was a single-arm phase II trial including patients with disease progression on or after endocrine therapy, with prior exposure to a taxane, and with at least 2 prior chemotherapy regimens including at least 1 in the metastatic setting. The objective response rate (ORR) was $19.7 \%$ (95\% CI [confidence interval], 13.3, 27.5), and the median OS was 22.3 months [15]. Although this was a single-arm study, the ORR and OS observed in MONARCH 1 suggest single-agent abemaciclib may offer a more favorable benefit-risk profile than what might be expected in patients receiving cytotoxic chemotherapy $[6,8]$. At the time of the MONARCH 1 trial, treatment options for this patient population were typically limited to chemotherapy [15]. However, without a comparator arm, it is difficult to put these findings into clinical context relative to available treatment options.

Traditionally, historical controls from previous clinical trials have been used to provide context for results from single-arm trials $[16,17]$, but this approach may be hampered by differing patient populations or lines of treatment. In contrast, real-world data (RWD) allow for selection of a more contemporaneous cohort of patients who match relevant trial criteria and patient-level data, and allow for matching between real-world and trial cohorts to balance patient characteristics [16, 18, 19]. This retrospective cohort study used RWD from patients treated in a clinical practice setting for $\mathrm{MBC}$ to create a single-agent chemotherapy control arm to help contextualize the results observed in MONARCH 1.

\section{Materials and methods}

\section{Data source}

This retrospective study utilized the Flatiron Health electronic health records (EHR)-derived database and included patients diagnosed with MBC from 01 January, 2011, to 28 February, 2018. The Flatiron Health database is a US-based longitudinal, demographically and geographically diverse database derived from EHR data from over 280 cancer clinics ( $~ 800$ sites of care) representing more than 2.1 million active cancer patients. As of March 2018, the Flatiron MBC cohort included more than 15,000 patients with MBC from approximately 180 clinics. Patients were included in the cohort if they were stage IV at initial diagnosis or if they developed recurrent $\mathrm{MBC}$ after an initial diagnosis of early stage breast cancer. The database includes both structured and some unstructured EHR data elements, such as patient demographics (gender, race, birth year, and state of residence), type of cancer facility visited (community vs. academic), clinical diagnoses, laboratory data, biomarker tests and results, medications ordered and/or administered, line of therapy (derived), month and year of death, and other patient clinical characteristics including cancer stage at initial diagnosis and performance status (PS). Date of death was derived from EHR data, commercial death data, and the Social Security Death Index [20]. 


\section{Real-world chemotherapy cohort key inclusion and exclusion criteria}

The inclusion and exclusion criteria applied to the Flatiron Health MBC Cohort for this study were intended to recapitulate the MONARCH 1 trial key eligibility criteria [15], where it was feasible to do so, in order to provide the most relevant real-world comparator to the MONARCH 1 patient population (Table 1). The criteria consisted of female patients with a diagnosis of HR+, HER2- MBC who received monotherapy with capecitabine, gemcitabine, eribulin, or vinorelbine in second or later lines of therapy. These agents were selected based on ESMO and NCCN guidelines for single-agent chemotherapy for patients in this setting.

Patients received at least 1 but no more than 2 lines of chemotherapy for those in the advanced setting prior to receipt of the single-agent chemotherapies listed above. Those included had an Eastern Cooperative Oncology Group (ECOG) performance status of 0 or 1 (within 60 days before or 30 days after the eligible line of therapy). Patients who received CDK4 \& 6 inhibitor drugs (palbociclib, ribociclib, and/or abemaciclib) in prior lines of therapy were excluded. Patients who received prior HER2 targeted therapy were excluded. Patients with diagnosis codes for central nervous system (CNS) metastasis (ICD 9: 198.3 and 198.4; ICD 10: C79.31, C79.32, and C79.49) on or before the eligible line of therapy were excluded.

Due to limited information within the data on the adjuvant setting, prior taxane and endocrine therapy in the adjuvant and/or metastatic setting were not required. Patients who received monotherapy with capecitabine, gemcitabine, eribulin, or vinorelbine in 1 or more lines of therapy qualified as eligible based on the above selection criteria. If a patient had only 1 eligible line of therapy, the index drug was the drug contained in the eligible line and the index date was the start date of the eligible line. If the patient had multiple eligible lines of therapy, a line was randomly selected from eligible lines and the index date was the start date of the randomly selected line of therapy (Table 2). The patients were followed from the index date until the date of death, loss to follow-up, or end of the database. The index date must have occurred at least 3 months prior to the end of the database.

\section{Statistical analyses}

Descriptive statistics were used to summarize baseline demographic and clinical characteristics for the MONARCH 1 and real-world chemotherapy cohorts. The primary endpoint of this exploratory analysis was OS, defined as the time from index date to either censoring or death. Patients without a date of death were censored at the last activity date. OS was analyzed by the Kaplan-Meier method and Cox proportional hazards regression in the matched cohorts. As the primary analysis, the Mahalanobis distance matching method [21] was used to match each patient from MONARCH 1 with a patient from the realworld chemotherapy cohort according to key baseline and disease characteristics. The following characteristics were incorporated into the matching process: age group, race group, number of prior chemotherapies in the metastatic setting, number of prior endocrine therapies in the metastatic setting, prior capecitabine use, and progesterone receptor status. The intent of the matching procedure is to

Table 1 Inclusion criteria for MONARCH 1 and real-world chemotherapy cohort

\begin{tabular}{lll}
\hline Key features & MONARCH 1 & Real-world cohort $^{\mathrm{a}}$ \\
\hline Indication & MBC & MBC \\
HR status & Positive & Positive \\
HER2 status & Negative & Negative \\
Prior endocrine therapy in adjuvant and/or metastatic setting & Yes & $\begin{array}{c}\text { Not required }{ }^{\text {a }} \text { but prior ET in meta- } \\
\text { static disease explored in sensitivity } \\
\text { analyses }\end{array}$ \\
Number of prior chemotherapy regimens in metastatic setting & & 1 or 2 \\
ECOG PS & 1 or 2 & 0 or 1 \\
Prior taxane containing regimen in adjuvant or metastatic setting & 0 or 1 & Not required \\
Prior CDK4 \& 6 therapy & Yes & Not permitted \\
CNS metastases & Not permitted & Not permitted \\
\hline
\end{tabular}

$C D K$ Cyclin-dependent kinase, $C N S$ central nervous system, ECOG PS eastern cooperative oncology group performance status, $E H R$ electronic health record, $E T$ endocrine therapy, HER2 human epidermal growth factor receptor 2, $H R$ hormone receptor, $M B C$ metastatic breast cancer

${ }^{a}$ Analyses were conducted both requiring and not requiring (primary analysis) prior endocrine therapy in the metastatic setting

'Prior taxane use was 'not required' in the real-world chemotherapy cohort because the Flatiron Health EHR database has incomplete data in the adjuvant setting 
Table 2 Attrition table for real-world chemotherapy cohort

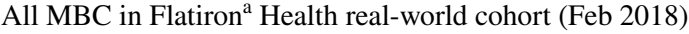

$C D K$ Cyclin-dependent kinase, $C N S$ central nervous system, ECOG PS eastern cooperative oncology group performance status, $E H R$ electronic health record, $E R$ estrogen receptor, $H E R 2$ human epidermal growth factor receptor 2, $M B C$ metastatic breast cancer, $N$ total number of patients, $P R$ progesterone receptor

${ }^{a}$ Flatiron Health EHR database (https://flatiron.com/real-world-evidence/) $022018^{*}$

${ }^{b}$ Eligible line contains any single-agent treatment (capecitabine, gemcitabine, eribulin, and vinorelbine) (a) received 1-2 lines of therapy containing chemotherapy drug prior to eligible line, (b) had a positive test for ER or positive test for PR on or before the eligible line, (c) had a negative test for HER2 on or before the line containing single-agent treatment, (d) had an ECOG PS $\leq 1$ (60 day window prior or 30 days after), (e) no diagnosis codes for CNS metastasis on or before the eligible line, (f) eligible line occurs $\geq 3$ months prior to end of database, (g) no CDK4 \& 6 inhibitor and no clinical study drug prior to the eligible line

*Date of most recent dataset utilized in the analyses, Feb 2018, mortality v2.0

correct for any observed imbalance due to differing baseline demographics and disease characteristics.

As a sensitivity analysis, entropy balancing was performed. Entropy balancing [22] provided a reweighting scheme used to adjust inequalities in distribution of baseline characteristics across the MONARCH 1 and realworld chemotherapy cohorts using pre-specified variables for reweighting. Variables for reweighting using entropy balancing included age group, race group, number of prior chemotherapy regimens in the metastatic setting, number of prior endocrine therapies in the metastatic setting, progesterone receptor status, prior capecitabine use, ECOG PS, and stage at initial diagnosis. The weighted Kaplan-Meier method was applied to the weighted realworld chemotherapy cohort to estimate median OS, and the bootstrap approach was used to estimate $95 \%$ confidence interval (CI) of the median OS.

The treatment effect was also evaluated among 2 subsets in additional sensitivity analyses. Since Flatiron Health data are US-based, the first subset was among US patients only, where treatment effect was evaluated between the entire real-world cohort and MONARCH 1 US patients. The second subset was US patients with prior endocrine therapy in the metastatic setting, where treatment effect was evaluated between the real-world cohort with prior endocrine therapy in the metastatic setting and the MONARCH 1 US patients with prior endocrine therapy in the metastatic setting to try and account for the prior endocrine therapy inclusion criterion within the trial.

Duration of therapy was defined as time from index date to last order or administration of the eligible drug during the line of therapy. If the drug was an oral medication, then 30 days were added to the last order date to assume a 30-day supply. Quartiles and median duration of treatment along with 95\% CIs were estimated using the Kaplan-Meier method. The Cox model was used to estimate HR. All hypothesis tests were conducted at a 2-sided alpha level of 0.05 , unless otherwise stated. All CIs were given at a 2 -sided 95\% level, unless otherwise stated. Data were analyzed using SAS version 9.2 or later (SAS Institute Inc.) and R (The R Foundation).

\section{Results}

After applying the inclusion and exclusion criteria, 281 patients were identified who received an eligible line of therapy and were included in the real-world chemotherapy cohort (Table 2).

\section{Baseline demographics and clinical characteristics}

Compared to the MONARCH 1 cohort $(n=132)$, the realworld cohort $(n=281)$ was more likely to be 65 years or older (43.4\% vs. $31.8 \%, p=0.03)$ and less likely to be white $(68.3 \%$ vs. $93.9 \%, p<0.0001)$ (Table 3$)$. A majority of the patients in MONARCH 1 (70 patients, 53.0\%) were enrolled at sites in the US. Unlike the real-world chemotherapy cohort which included all US patients, patients in MONARCH 1 were also enrolled at sites in Belgium (28 patients, 21.2\%), Spain (23 patients, $17.4 \%$ ), and France (11 patients, $8.3 \%)$. Only 17 (12.9\%) patients in the MONARCH 1 cohort had not received prior endocrine therapy in the metastatic setting compared to $114(40.6 \%)$ patients in the real-world chemotherapy cohort. More than half $(76,57.6 \%)$ had prior capecitabine in the MONARCH 1 cohort compared to only $73(26.0 \%)$ in the real-world chemotherapy cohort (Table 3). The cohorts were similar with respect to number of prior 
Table 3 Selected baseline characteristics: before and after Mahalanobis distance matching

\begin{tabular}{|c|c|c|c|c|c|c|}
\hline \multirow[t]{2}{*}{ Factors } & \multicolumn{3}{|l|}{ Before matching } & \multicolumn{3}{|l|}{ After matching } \\
\hline & $\begin{array}{l}\text { MONARCH } 1 \\
(N=132)\end{array}$ & $\begin{array}{l}\text { Real-world cohort } \\
(N=281)\end{array}$ & $p$-value & $\begin{array}{l}\text { MONARCH } 1 \\
(N=108)\end{array}$ & $\begin{array}{l}\text { Real-world cohort } \\
(N=108)\end{array}$ & $p$-value \\
\hline Pooled age Group, $n(\%)$ & & & 0.031 & & & 1.0 \\
\hline$<65$ years & $90(68.2)$ & $159(56.6)$ & & $72(66.7)$ & $71(65.7)$ & \\
\hline$\geq 65$ years & $42(31.8)$ & $122(43.4)$ & & $36(33.3)$ & $37(34.3)$ & \\
\hline Pooled race group, $n(\%)$ & & & $<.0001$ & & & 0.569 \\
\hline Other & $8(6.1)$ & $89(31.7)$ & & $8(7.4)$ & $5(4.6)$ & \\
\hline White & $124(93.9)$ & $192(68.3)$ & & $100(92.6)$ & $103(95.4)$ & \\
\hline Lines of chemotherapy ${ }^{\mathrm{a}}, n(\%)$ & & & 0.290 & & & 0.783 \\
\hline 1 Regimen & $67(50.8)$ & $159(56.6)$ & & $61(56.5)$ & $64(59.3)$ & \\
\hline 2 Regimens & $65(49.2)$ & $122(43.4)$ & & $47(43.5)$ & $44(40.7)$ & \\
\hline Lines of prior $\mathrm{ET}^{\mathrm{a}}, n(\%)$ & & & $<.0001$ & & & 0.984 \\
\hline 0 Regimen & $17(12.9)$ & $114(40.6)$ & & $17(15.7)$ & $16(14.8)$ & \\
\hline 1 Regimen & $48(36.4)$ & $77(27.4)$ & & $40(37.0)$ & $42(38.9)$ & \\
\hline 2 Regimens & $25(18.9)$ & $54(19.2)$ & & $23(21.3)$ & $24(22.2)$ & \\
\hline $3+$ Regimens & $42(31.8)$ & $36(12.8)$ & & $28(25.9)$ & $26(24.1)$ & \\
\hline PgR Status & & & & & & 0.879 \\
\hline Negative & $35(26.5)$ & $99(35.2)$ & .090 & $29(26.9)$ & $31(28.7)$ & \\
\hline Positive & $95(72.0)$ & $179(63.7)$ & & $79(73.1)$ & $77(71.3)$ & \\
\hline Prior capecitabine use, $n(\%)$ & & & $<.0001$ & & & 0.586 \\
\hline No & $56(42.4)$ & $208(74.0)$ & & $54(50.0)$ & $59(54.6)$ & \\
\hline Yes & $76(57.6)$ & 73 (26.0) & & $54(50.0)$ & $49(45.4)$ & \\
\hline
\end{tabular}

Fisher's exact test was used for $p$-value

Patients with missing baseline disease characteristics were removed from the matching protocol

$E T$ Endocrine therapy, $N$ total number of patients, $n$ number of patients within a specific category, $P g R$ progesterone receptor

${ }^{a}$ In metastatic setting

lines of chemotherapy in the metastatic setting and progesterone receptor status.

The Mahalanobis distance matching method was used to select patients who had the shortest Mahalanobis distances from the 132 patients in MONARCH 1 and the 281 patients in the real-world chemotherapy cohort. Patients with missing baseline disease characteristics were removed from the distance calculation. Following Mahalanobis distance matching, 108 patients from the MONARCH 1 cohort were matched to 108 patients from the real-world chemotherapy cohort, and the cohorts had similar patient and disease characteristics. In the matched cohorts, no statistically significant differences existed in age, race, lines of chemotherapy in the metastatic setting, prior endocrine therapy in the metastatic setting, progesterone receptor status, and prior capecitabine use (Table 3). The prior therapy profile between the matched MONARCH 1 and real-world chemotherapy cohort was also similar (Table 4). In the MONARCH 1 cohort there was greater tamoxifen $(29,26.9 \%$ vs. $12,11.1 \%)$, letrozole (40, $37.0 \%$ vs. $20,18.5 \%)$, and bevacizumab $(13,12.0 \%$ vs. 0$)$ use compared to the real-world chemotherapy cohort in the metastatic setting (Table 4).

\section{Overall survival}

Using the matching method, the adjusted median OS was 22.3 months (95\% CI: 16.0, NR) in MONARCH 1 and 13.6 months $(95 \%$ CI: 9.6, 16.6) in the real-world chemotherapy cohort. The estimated HR between the 2 matched adjusted groups was 0.536 (95\% CI: 0.37, 0.77) (Table 5, Fig. 1).

The results of sensitivity analyses, using entropy balancing as a secondary analytic approach, were consistent with the Mahalanobis distance matching results. The adjusted median OS was 12.7 months in the real-world cohort $(n=281)$ with a HR of $0.56(95 \%$ CI from bootstrapping: $0.44,0.78)$. The sensitivity analysis in the realworld chemotherapy cohort $(n=281)$ and MONARCH 1 US patients only $(n=70)$ was consistent with a HR of 0.60 (95\% CI: $0.45,0.93$ ). The sensitivity analysis of the patients with prior endocrine therapy for $\mathrm{MBC}$ in the realworld chemotherapy cohort $(n=167)$ and in the MONARCH 1 US cohort $(n=62)$ was also consistent with a HR of 0.52 (95\% CI: 0.37, 0.82) (Fig. 2). 
Table 4 Prior therapy comparison between matched MONARCH 1 and real-world chemotherapy cohort

\begin{tabular}{|c|c|c|}
\hline Patients with any prior therapy & $\begin{array}{l}\text { Monarch } 1 \\
N=108\end{array}$ & $\begin{array}{l}\text { Real-world cohort } \\
N=108\end{array}$ \\
\hline Chemotherapy ${ }^{\mathrm{a}}, \mathrm{n}(\%)$ & $108(100)$ & $108(100)$ \\
\hline Paclitaxel & $55(50.9)$ & $50(46.3)$ \\
\hline Capecitabine & $51(47.2)$ & $49(45.4)$ \\
\hline Docetaxel & $18(16.7)$ & $11(10.2)$ \\
\hline Cyclophosphamide & $13(12.0)$ & $7(6.5)$ \\
\hline Gemcitabine & $10(9.3)$ & $15(13.9)$ \\
\hline Eribulin & $6(5.6)$ & $7(6.5)$ \\
\hline Other $^{b}$ & $34(31.5)$ & $34(31.5)$ \\
\hline Endocrine therapy ${ }^{\mathrm{a}}, n(\%)$ & $91(84.3)$ & $92(85.2)$ \\
\hline Fulvestrant & $52(48.1)$ & $59(54.6)$ \\
\hline Exemestane & $45(41.7)$ & $42(38.9)$ \\
\hline Letrozole & $40(37.0)$ & $20(18.5)$ \\
\hline Tamoxifen & $29(26.9)$ & $12(11.1)$ \\
\hline Anastrozole & $19(17.6)$ & $22(20.4)$ \\
\hline Other $^{\mathrm{c}}$ & $14(13.0)$ & $10(9.3)$ \\
\hline Targeted therapy ${ }^{\mathrm{a}}, n(\%)$ & $48(44.4)$ & $32(29.6)$ \\
\hline Everolimus & $29(26.9)$ & $32(29.6)$ \\
\hline Bevacizumab & $13(12.0)$ & 0 \\
\hline Other $^{\mathrm{d}}$ & $13(12.0)$ & $1(0.9)$ \\
\hline Other $^{\mathrm{a}}, n(\%)$ & $21(19.4)$ & $2(1.9)$ \\
\hline Investigational drug & $14(13.0)$ & 0 \\
\hline Other ${ }^{\mathrm{e}}$ & $10(9.3)$ & $2(1.9)$ \\
\hline
\end{tabular}

$N$ Total number of patients, $n$ number of patients within a specific category

${ }^{a}$ Any single therapy with $>10 \%$ in either arm is listed; all other therapies, except eribulin (included in the table) are combined into 'other' in each category

${ }^{\mathrm{b}}$ Other chemotherapy agents include doxorubicin, carboplatin, fluorouracil, vinorelbine, epirubicin, cyclophosphamide w/epirubicin hydrochloride/f, cyclophosphamide w/doxorubicin, methotrexate, mitoxantrone, oxaliplatin, paclitaxel w/carboplatin, cisplatin, doxorubicin pegylated liposomal, and ixabepilone

${ }^{\mathrm{c}}$ Other endocrine therapy regimens include megestrol, abiraterone, bicalutamide, diethylstilbestrol, enzalutamide, gonadorelin, goserelin, leuprorelin, medroxyprogesterone, toremifene, triptorelin, and leuprolide

${ }^{\mathrm{d}}$ Other targeted therapies include dasatinib, ganetespib, taselisib, abexinostat, buparlisib, olaparib, ramucirumab, ridaforolimus, ruxolitinib, veliparib, and cetuximab

${ }^{\mathrm{e}}$ Other other therapies include denosumab, zoledronic acid, fluoxymesterone, prednisolone, leucovorin, and sorafenib

\section{Duration of therapy}

Duration of therapy was not significantly different between the 2 cohorts. The median duration of therapy was 4.1 months in MONARCH 1 compared to 2.9 months in the real-world chemotherapy cohort $(p=0.050)$ with a HR of 0.764 (95\% CI: 0.58, 1.00) (Table 6).

\section{Post-discontinuation therapy}

Post-discontinuation therapy was consistent between the 2 cohorts. In total, 71 patients in the real-world chemotherapy cohort received at least 1 subsequent therapy compared with 77 patients in MONARCH 1. A total of 61 patients in the real-world cohort received chemotherapy as any post-discontinuation therapy compared with 65 patients in MONARCH 1. However, more patients in the real-world chemotherapy cohort received palbociclib $(n=32)$ or endocrine therapy $(n=50)$ versus patients in MONARCH $1(n=0$ receiving palbociclib, $n=13$ receiving endocrine therapy) as any postdiscontinuation therapy (Table 7).

\section{Discussion}

Abemaciclib has demonstrated clinical activity as a monotherapy in patients with HR+, HER2- MBC heavily pretreated in the advanced setting with both endocrine and chemotherapy in MONARCH 1 (NCT02102490) [15]. Here, we compared OS and duration of treatment in MONARCH 1 with a matched real-world cohort of patients who received standard-of-care treatment with single-agent chemotherapy and had not previously received CDK4 \& 6 inhibitors using the Flatiron database. Although MONARCH 1 did not include a control arm, this real-world chemotherapy cohort provided a data source representative of a control arm. The OS was significantly longer in the MONARCH 1 cohort at 22.3 months compared to 13.6 months in the matched real-world chemotherapy cohort. Duration of treatment was not significantly different between the two groups; however, it was numerically longer in the MONARCH 1 cohort (4.1 months) compared to the real-world cohort (2.9 months). Altogether, these results suggest a possible survival advantage in favor of abemaciclib as a monotherapy in these patients.

In certain cases, regulatory agencies have accepted the use of real-world control arms to contextualize results from single-arm trials to support regulatory decisions. The increasing accessibility of digital health data, in combination with rising costs and recognized limits of traditional trials, has renewed interest in the use of RWD to enhance the efficiency of research and bridge the evidentiary gap between clinical research and practice [23]. The FDA Real-World Evidence (RWE) Framework notes RWE can be used as the basis for external controls in some situations [16]. Furthermore, RWE has been pivotal in some European regulatory decisions involving conditions with significant unmet need and when a randomized clinical 
Table 5 Overall survival between matched MONARCH 1 and real-world chemotherapy cohort
Fig. 1 Overall survival between matched MONARCH 1 and real-world chemotherapy cohort. $p$-value (2-sided) LOGRANK unstratified for comparing MONARCH 1 with real-world chemotherapy cohort. CI Confidence interval, $H R$ hazard ratio, $N R$ not recorded

\begin{tabular}{llll}
\hline & $\begin{array}{l}\text { Monarch 1 } \\
N=108\end{array}$ & $\begin{array}{l}\text { Real-world cohort } \\
N=108\end{array}$ & Difference/ $p$-value \\
\hline Number of deaths, $n(\%)$ & $50(46.3)$ & $79(73.1)$ & \\
Patients censored, $n(\%)$ & $58(53.7)$ & $29(26.9)$ & \\
No documented deaths & $58(53.7)$ & $29(26.9)$ & \\
Survival rate, \% (95\% CI) & & & \\
4-month & $91.7(84.6,95.6)$ & $85.1(76.9,90.6)$ & $6.5(-2.0,15.0) / p=0.133$ \\
8-month & $85.1(76.9,90.6)$ & $66.1(56.2,74.2)$ & $19.0(7.8,30.3) / p=0.0009$ \\
12-month & $72.7(63.2,80.2)$ & $55.2(45.1,64.1)$ & $17.5(4.8,30.3) / p=0.007$ \\
24-month & $49.1(38.1,59.2)$ & $28.5(19.5,38.1)$ & $20.6(6.4,34.8) / p=0.004$ \\
\hline
\end{tabular}

Overall survival rates were estimated using the Kaplan-Meier method. Corresponding 95\% CIs were estimated using the methods of Brookmeyer and Crowley, and Greenwood, respectively

$C I$ Confidence interval, $N$ total number of patients, $n$ number of patients within a specific category

${ }^{\mathrm{a}} 95 \% \mathrm{CIs}$ and 2-sided $p$-values for the difference between rates were calculated based on normal approximation

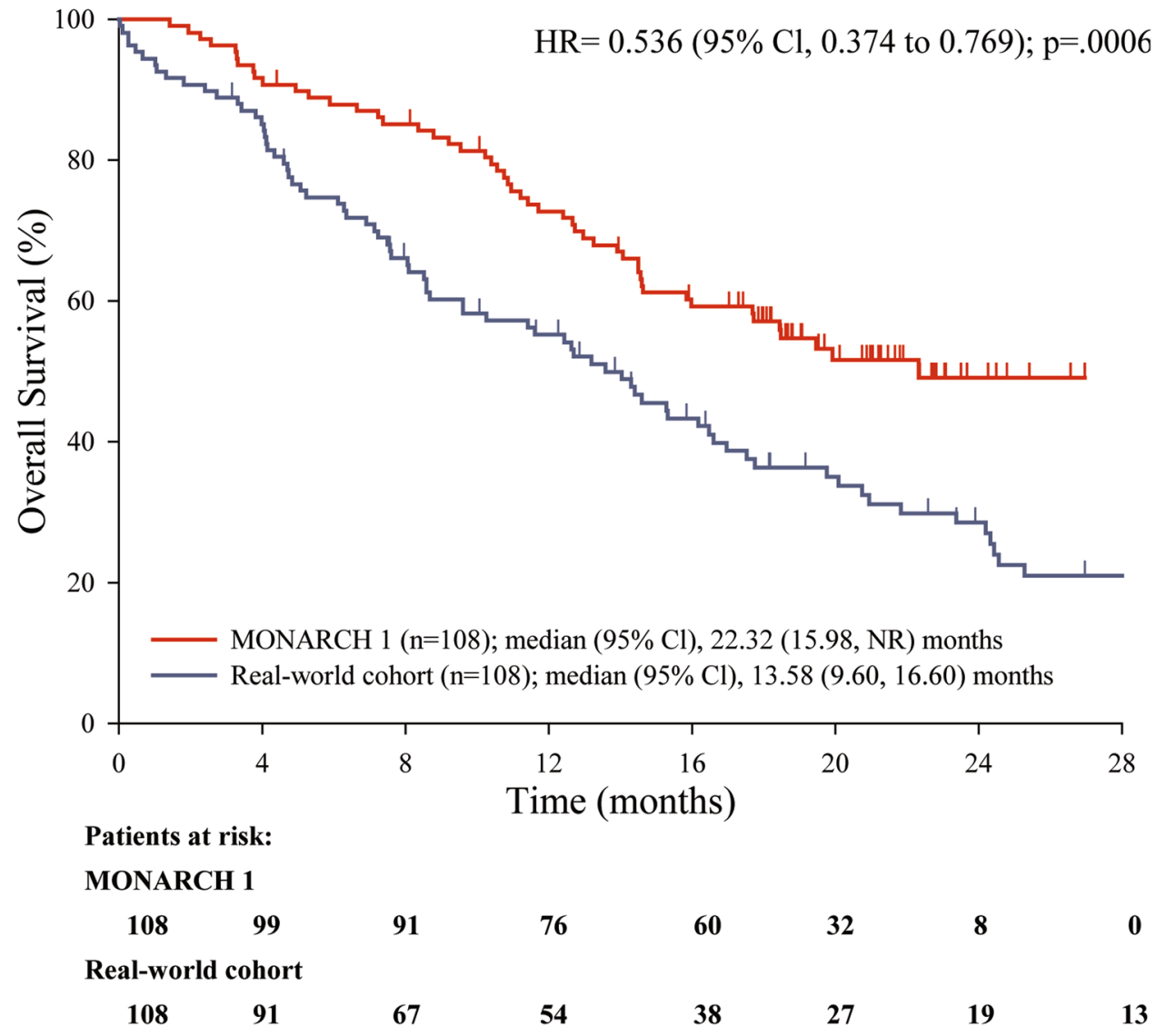

trial is unfeasible or unethical [24]. For example, in the case of blinatumomab, the real-world cohort was helpful in supporting accelerated approval for the treatment of acute lymphoblastic leukemia by the European Medicines Agency [25, 26]. In the case of avelumab as a monotherapy for metastatic Merkel cell carcinoma (mMCC), RWE was used to characterize the natural history of mMCC and was offered to regulators as a benchmark. A subset of trial patients who responded well to treatment was identified and the benefit documented through contrast with the RWE benchmark data, leading to regulatory approval in the US, European Union, and Japan for that subset [19, $27,28]$.

There have been improvements in the quality of data over the past several years, with access to recent data containing rich clinical variables and relevant endpoints. For 
Fig. 2 Sensitivity analyses of overall survival. ${ }^{a}$ Method in main analysis. ${ }^{\mathrm{b}}$ Entropy balancing method was applied. Sample sizes are as follows: Mahalanobis distance method: real-world chemotherapy cohort $(n=108)$ vs. MONARCH $1(n=108)$. Entropy balancing: real-world chemotherapy cohort $(n=281)$ vs. MONARCH $1(n=132)$. US patients only: real-world chemotherapy cohort $(n=281)$ vs. US patients only $(n=70)$. US patients who received prior ET: real-world chemotherapy cohort with prior ET $(n=167)$ vs. US patients who received prior ET in metastatic setting $(\mathrm{n}=62)$. ET endocrine therapy

Table 6 Duration of therapy

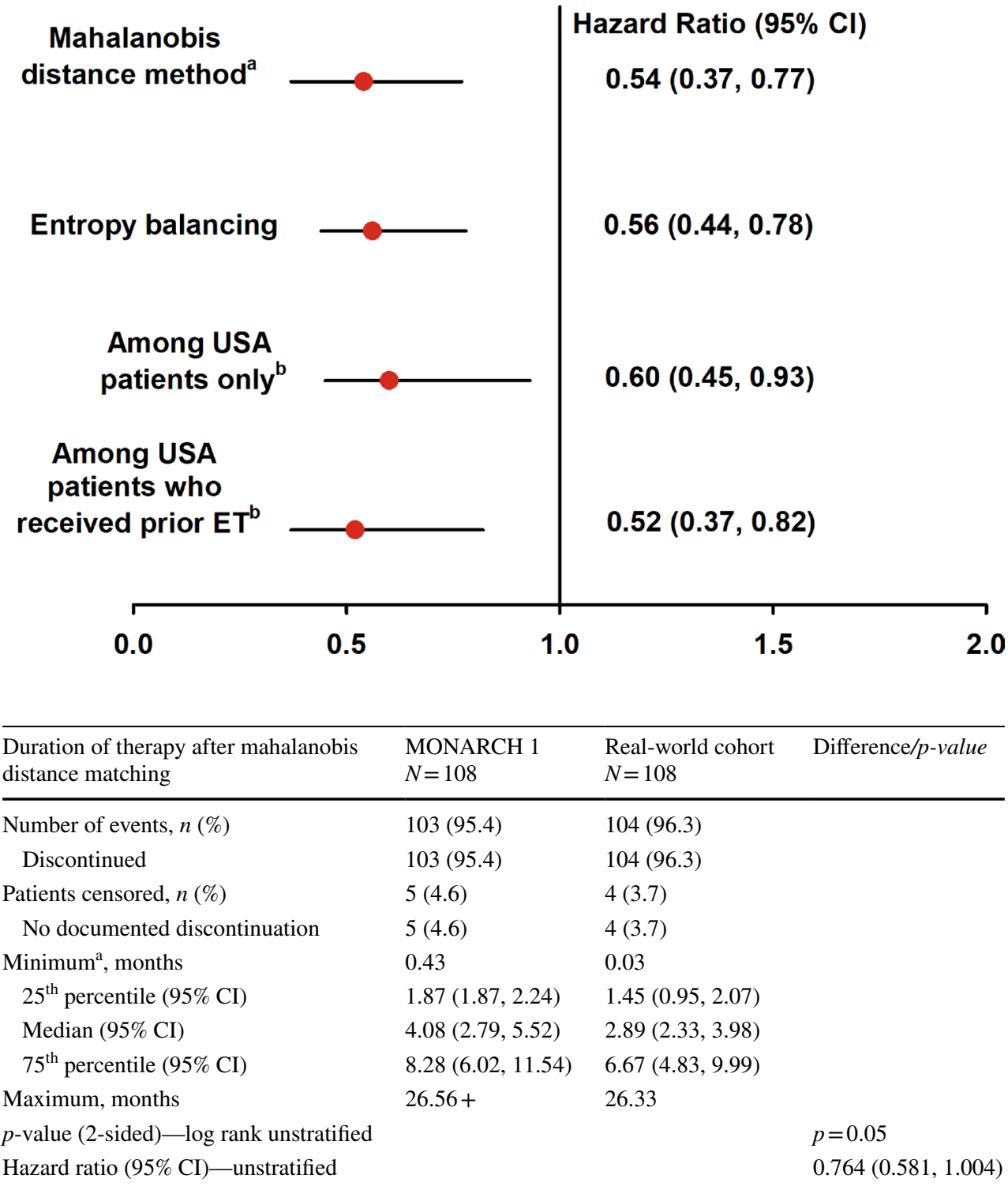

Quartiles and duration of treatment survival rates, along with 95\% CIs were estimated using the KaplanMeier method. Corresponding 95\% CIs were estimated using the methods of Brookmeyer and Crowley, and Greenwood, respectively

$C I$ Confidence interval, $N$ total number of patients, $n$ number of patients within a specific category

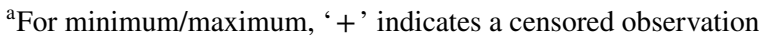

example, the Flatiron Health EHR-derived database represents a diverse group of community cancer clinics, ranging from small practices to large multicenter practices. A full copy of each patient's medical record is pulled into a central repository for processing. Structured data such as demographics, medications, and routine laboratory tests are harmonized and normalized to a standard ontology and common data model. These structured data are processed and harmonized centrally by Flatiron's technology-assisted data engine and made accessible for research and analytics. Unstructured data such as case notes, pathology reports, and complex laboratory tests are turned into discrete analyzable data using technology driven abstraction [29, 30].

We attempted to mimic the eligibility criteria for MONARCH 1 in the Flatiron cohort, and we used matching and balancing methods to control for differences in key measured confounders between cohorts. To adjust for potential differences, the Mahalanobis distance matching method [21] was used to match each patient from MONARCH 1 with a patient from the real-world chemotherapy cohort. The aim of this method was to select a subset of patients from the real-world chemotherapy cohort with the most comparable baseline and disease characteristic to the MONARCH 1 
Table 7 Summary of postdiscontinuation treatment

\begin{tabular}{|c|c|c|}
\hline $\begin{array}{l}\text { Any post-discontinuation therapy after mahalanobis distance } \\
\text { matching }\end{array}$ & $\begin{array}{l}\text { MONARCH } 1 \\
N=108\end{array}$ & $\begin{array}{l}\text { Real-world cohort } \\
N=108\end{array}$ \\
\hline Patients with any post-discontinuation therapy, $n(\%)$ & $77(71.3)$ & $71(65.7)$ \\
\hline Chemotherapy $^{\mathrm{a}}, n(\%)$ & $65(60.2)$ & $61(56.5)$ \\
\hline Capecitabine & $26(24.1)$ & $14(13.0)$ \\
\hline Eribulin & $18(16.7)$ & $18(16.7)$ \\
\hline Doxorubicin & $16(14.8)$ & $3(2.8)$ \\
\hline Paclitaxel & $15(13.9)$ & $16(14.8)$ \\
\hline Vinorelbine & $13(12.0$ & $11(10.2)$ \\
\hline Gemcitabine & $3(2.8)$ & $17(15.7)$ \\
\hline Doxorubicin pegylated liposomal & 0 & $19(17.6)$ \\
\hline Other $^{b}$ & $18(16.7)$ & $50(46.3)$ \\
\hline Endocrine therapy ${ }^{\mathrm{c}}, n(\%)$ & $13(12.0)$ & $50^{\mathrm{d}}(46.3)$ \\
\hline Fulvestrant & $6(5.6)$ & $17(15.7)$ \\
\hline Exemestane & $3(2.8)$ & $15(13.9)$ \\
\hline Letrozole & $3(2.8)$ & $26(24.1)$ \\
\hline Other ${ }^{\mathrm{e}}$ & $4(3.7)$ & $9(8.3)$ \\
\hline Targeted therapy, $n(\%)$ & $8(7.4)$ & $41(38.0)$ \\
\hline Everolimus & $5(4.6)$ & $10(9.3)$ \\
\hline Palbociclib & 0 & $32(29.6)$ \\
\hline Other $^{\mathrm{f}}$ & $3(2.8)$ & $5(4.6)$ \\
\hline Other, $n(\%)$ & $8(7.4)$ & $6(5.6)$ \\
\hline Investigational drug & $6(5.6)$ & $4(3.7)$ \\
\hline Other $^{g}$ & $2(1.9)$ & $2(1.9)$ \\
\hline
\end{tabular}

$C D K$ Cyclin-dependent kinases, $N$ total number of patients, $n$ number of patients within a specific category aAny single chemotherapy agent with $>10 \%$ in either arm is listed; all other therapies are combined into 'other'

${ }^{b}$ Other chemotherapy agents include cyclophosphamide, fluorouracil, docetaxel, cisplatin w/fluorouracil, cyclophosphamide w/epirubicin hydrochloride/f, cyclophosphamide w/fluorouracil/methotrexate, epirubicin, lurbinectedin, methotrexate, thiotepa, carboplatin, cisplatin, etoposide, irinotecan, and ixabepilone

${ }^{c}$ Any endocrine, targeted, or other therapy with $>5 \%$ in either arm is listed; all other therapies are combined into 'other'

d 32 out of 50 patients who received endocrine therapy also received concurrent CDK4 \& 6 inhibitor

${ }^{\mathrm{e}}$ Other endocrine therapy regimens include tamoxifen, orteronel, anastrozole, and leuprolide

${ }^{\mathrm{f}}$ Other targeted therapies include bevacizumab, cabozantinib, trastuzumab, nivolumab, and ribociclib

${ }^{g}$ Other therapies include dexrazoxane, doxycycline, and leucovorin population. Following this procedure, known and measured baseline characteristics were balanced between the cohorts. Any observed imbalance due to differing baseline and disease characteristics was corrected, as illustrated by the lack of significance in any of the $p$-values.

Some of the potential limitations to this approach have been addressed through sensitivity analyses. However, we were unable to match the trial eligibility criteria exactly due to a lack of data availability in Flatiron (such as incomplete data on adjuvant therapy) and unmeasured confounders. Data on adjuvant therapy, including prior taxane use, are not complete in the real-world chemotherapy cohort because patients may have received care prior to adoption of an EHR at the practice, or patients may have received care at a practice outside the Flatiron Health network. Other variables such as sites of metastatic disease (e.g. visceral disease or liver metastases), previous cancers, and comorbidities were not included in the Flatiron Health data. Therefore, residual differences between the real-world chemotherapy and MONARCH 1 cohorts may have contributed to the observed outcomes, even after matching on key measured variables. Another limitation is orders for oral therapies may be incomplete in the structured EHR data, as subsequent refills may not be documented, thus duration of treatment may have been underestimated in the real-world chemotherapy cohort. Another limitation inherent to real-world studies is the potential for selection bias. The fact that clinicians chose to give 1 cohort of patients' chemotherapy instead of endocrine or targeted therapy could imply patients in the chemotherapy only arm is a higher risk group of patients. 
Furthermore, the MONARCH 1 and real-world chemotherapy cohort were not contemporaneous. MONARCH 1 enrolled patients from June 2014 through April 2015, while the real-world chemotherapy cohort included patients with index dates from January 2011 through February 2018. As a result, $29.6 \%$ of patients in the real-world chemotherapy cohort received a CDK4 \& 6 inhibitor following discontinuation of the index therapy. This suggests the survival in the real-world cohort is potentially longer than it would have been in a truly contemporaneous cohort, thus the difference in overall survival observed in this analysis may be underestimated. Finally, MONARCH 1 included patients from Europe and the US, while the real-world chemotherapy cohort was from the US only. However, sensitivity analyses suggest results are consistent in the subset of MONARCH 1 patients from the US (Fig. 2).

\section{Overall conclusion}

Methodological advances in statistical analyses and improvements in data quality enable the use of a real-world single-agent chemotherapy cohort as an external comparator arm. This study demonstrated an approach to create a realworld chemotherapy cohort to serve as a suitable comparator for MONARCH 1. These exploratory results suggest a possible survival advantage in heavily pretreated patients with advanced MBC treated with abemaciclib monotherapy compared to those treated with single-agent chemotherapy.

Acknowledgements We thank all the patients and their caregivers for participation in the trial. We thank all the investigators and their support staff who generously participated in this work. This study was sponsored by Eli Lilly and Company. Medical writing support was provided by Tolu Makinde, $\mathrm{PhD}$ RAC, and Sarah Beckman, $\mathrm{PhD}$, and editorial support was provided by Dana Schamberger, MA, and Cynthia Abbott of Syneos Health and funded by Eli Lilly and Company.

Funding This study was funded by Eli Lilly and Company.

Data availability Eli Lilly and Company provides access to all individual participant data collected during the trial, after anonymization, with the exception of pharmacokinetic or genetic data. Data are available to request 6 months after the indication studied has been approved in the United States and European Union and after primary publication acceptance, whichever is later. No expiration date of data requests is currently set once data are made available. Access is provided after a proposal has been approved by an independent review committee identified for this purpose and after receipt of a signed data sharing agreement. Data and documents (including the study protocol, statistical analysis plan, clinical study report, and blank or annotated case report forms) will be provided in a secure data sharing environment. For details on submitting a request, see the instructions provided at www.vivli.org.

\section{Compliance with ethical standards}

Conflict of interest Hope Rugo received grants from Pfizer, Novartis, Eli Lilly and Company, Genentech/Roche, Macrogenics, OBI, Merck, Eisai, Immunomedics, Daiichi Sankyo, Seattle Genetics, and Odonate. She received travel support from Daiichi, Mylan, Pfizer, Merck, AstraZeneca, Novartis, Macrogenics, and is a consultant to Samsung and Puma. Sara M. Tolaney is a consultant/advisor for AstraZeneca, Eli Lilly and Company, Merck, Nektar, Novartis, Pfizer, Genentech/ Roche, Immunomedics, Bristol Myers Squibb, Eisai, Nanostring, Puma, Sanofi, Celldex, Paxman, Odonate, Seattle Genetics, Silverback Therapeutics, G1 Therapeutics, AbbVie, Anthenex, OncoPep. She has received travel support from Astra Zeneca, Eli Lilly and Company, Merck, Nektar, Novartis, Pfizer, Genetech/Roche, Eisai, Immunomedics, Nanostring, Puma. Her institution received funding from Astra Zeneca, Merck, Nektar, Novartis, Pfizer, Genetech/Roche, Immunomedics, Exelixis, Eisai, Nanostring, Cyclacel, Sanofi, Odonate, Seattle Genetics. Javier Cortes is a consultant/advisor to Roche, Celgene, Cellestia, AstraZeneca, Biothera Pharmaceutical, Merus, Seattle Genetics, Daiichi Sankyo, Erytech, Athenex, Polyphor, Eli Lilly and Company, Servier, Merck Sharp \& Dohme, GSK, Leuko, Bioasis, Clovis Oncology, Boehringer Ingelheim. He has received honoraria from Roche, Novartis, Celgene, Eisai, Pfizer, Samsung Bioepis, Eli Lilly and Company, Merck Sharp \& Dohme, Daiichi Sankyo. His institution received research funding from Roche, Ariad Pharmaceuticals, AstraZeneca, Baxalta GMBH/Servier Affaires, Bayer Healthcare, Eisai, F.HoffmanLa Roche, Guardanth Health, Merck Sharp \& Dohme, Pfizer, Piqur Therapeutics, Puma C, and Queen Mary University of London. He owns stock, patents and intellectual property in MedSIR. He received travel support from Roche, Novartis, Eisai, Pfizer, and Daiichi Sankyo. Hans Wildiers received travel support from Roche and Pfizer. His institution received consulting fees and honoraria from AbbVie, Amgen, Ariez International, AstraZeneca, Biocartes, Celldex Therapeutics, DNA Prime, Janssen-CILAG, Eli Lilly and Company, Novartis, ORION Corporation, Pfizer, The Planning Shop, PUMA Biotechnology, Roche, Sirtex, TRM Oncology, and Vifor Pharma. His institution received an unrestricted research grant from Roche. Esther Zamora received personal fees from Roche and Novartis, and non-financial support from Eli Lilly and Company, Pfyzer and Roche. Veronique Dieras has served as an advisor to Eli Lilly and Company, Roche Genetech, Pfizer, Novartis AbbVie, Daiichi Sankyo, Astra Zeneca, Merk MSD France, and Seattle Genetics. Joyce O'Shaughnessy is a consultant/ advisor to AbbVie Inc., Agendia, Amgen Biotechnology, AstraZeneca, Bristol Myers Squibb, Celgene Corporation, Eisai, Genentech, Genomic Health, GRAIL, Immunomedics, Heron Therapeautics, Ipsen Biopharmaceuticals, Jounce Therapeutics, Eli Lilly and Company, Merck, Myriad, Novartis, Ondonate Therapeutics, Pfizer, Puma Biotechnology, Prime Oncology, Roche, Seattle Genetics, Syndax Pharmaceuticals, and Takeda. Denise Yardley is an advisor/consultant to Bristol Myers Squibb, Genetech, Immunomedics, Novartis, Odonate Therapeutics, R-Pharm US, Seattle Genetics. Her institution received funding from Genentech, Novartis, Odonate Therapeutics, AbbVie, Dana Farber Cancer Institute, Eli Lilly and Company, G1 Therapeutics, MacroGenics, Medivation, NSABP, Pfizer, US Oncology, Amgen, BIOMARIN, Biothera Pharmaceuticals, Clovis Pharma, Eisai, Incyte, Innocrin Pharmaceuticals, MedImmune, Merck, Merrimack Pharmaceuticals, Nektar Therapeutics, and Tesaro. Kristin M. Sheffield, Li Li, Yu-Jing Huang, Maura Dickler, Martin Frenzel, Xiaohong Ivy Li, Gebra Cuyun Carter are employees of Eli Lilly and Company and own stock in Eli Lilly and Company. Debra Patt has nothing to disclose.

Ethical approval All procedures performed in studies involving human participants were in accordance with the ethical standards of the insti- 
tutional and/or national research committee and with the 1964 Helsinki Declaration and its later amendments or comparable ethical standards.

Informed consent Informed consent was obtained from all individual participants included in the study.

Open Access This article is licensed under a Creative Commons Attribution 4.0 International License, which permits use, sharing, adaptation, distribution and reproduction in any medium or format, as long as you give appropriate credit to the original author(s) and the source, provide a link to the Creative Commons licence, and indicate if changes were made. The images or other third party material in this article are included in the article's Creative Commons licence, unless indicated otherwise in a credit line to the material. If material is not included in the article's Creative Commons licence and your intended use is not permitted by statutory regulation or exceeds the permitted use, you will need to obtain permission directly from the copyright holder. To view a copy of this licence, visit http://creativecommons.org/licenses/by/4.0/.

\section{References}

1. Howlader N, Altekruse SF, Li CI, Chen VW, Clarke CA, Ries LA, Cronin KA (2014) US incidence of breast cancer subtypes defined by joint hormone receptor and HER2 status. J Natl Cancer Inst. https://doi.org/10.1093/jnci/dju055

2. Cardoso F, Costa A, Norton L, Senkus E, Aapro M, Andre F, Barrios CH, Bergh J, Biganzoli L, Blackwell KL, Cardoso MJ, Cufer T, El Saghir N, Fallowfield L, Fenech D, Francis P, Gelmon K, Giordano SH, Gligorov J, Goldhirsch A, Harbeck N, Houssami N, Hudis C, Kaufman B, Krop I, Kyriakides S, Lin UN, Mayer M, Merjaver SD, Nordstrom EB, Pagani O, Partridge A, Penault-Llorca F, Piccart MJ, Rugo H, Sledge G, Thomssen C, Van't Veer L, Vorobiof D, Vrieling C, West N, Xu B, Winer E (2014) ESO-ESMO 2nd international consensus guidelines for advanced breast cancer (ABC2) ${ }^{\dagger}$. Ann Oncol 25:1871-1888. https ://doi.org/10.1093/annonc/mdu385

3. National Comprehensive Cancer Network (NCCN) (2018) Guidelines for patients (Metastatic Breast Cancer). https://www.nccn. org/patients/guidelines/stage_iv_breast/28/ Acccessed 23 July 2019

4. Cardoso F, Senkus E, Costa A, Papadopoulos E, Aapro M, Andre F, Harbeck N, Aguilar Lopez B, Barrios CH, Bergh J, Biganzoli L, Boers-Doets CB, Cardoso MJ, Carey LA, Cortes J, Curigliano G, Dieras V, El Saghir NS, Eniu A, Fallowfield L, Francis PA, Gelmon K, Johnston SRD, Kaufman B, Koppikar S, Krop IE, Mayer M, Nakigudde G, Offersen BV, Ohno S, Pagani O, Paluch-Shimon S, Penault-Llorca F, Prat A, Rugo HS, Sledge GW, Spence D, Thomssen C, Vorobiof DA, Xu B, Norton L, Winer EP (2018) 4th ESO-ESMO international consensus guidelines for advanced breast cancer (ABC 4) ${ }^{\dagger}$. Ann Oncol 29:1634-1657. https://doi.org/10.1093/annonc/mdy192

5. O'Shaughnessy J, Miles D, Vukelja S, Moiseyenko V, Ayoub JP, Cervantes G, Fumoleau P, Jones S, Lui WY, Mauriac L, Twelves C, Van Hazel G, Verma S, Leonard R (2002) Superior survival with capecitabine plus docetaxel combination therapy in anthracycline-pretreated patients with advanced breast cancer: phase III trial results. J Clin Oncol 20:2812-2823. https://doi.org/10.1200/ JCO.2002.09.002

6. Cortes J, O'Shaughnessy J, Loesch D, Blum JL, Vahdat LT, Petrakova K, Chollet P, Manikas A, Dieras V, Delozier T, Vladimirov V, Cardoso F, Koh H, Bougnoux P, Dutcus CE, Seegobin S, Mir D, Meneses N, Wanders J, Twelves C, investigators E (2011)
Eribulin monotherapy versus treatment of physician's choice in patients with metastatic breast cancer (EMBRACE): a phase 3 open-label randomised study. Lancet 377:914-923. https://doi. org/10.1016/S0140-6736(11)60070-6

7. Krop IE, Kim SB, Gonzalez-Martin A, LoRusso PM, Ferrero JM, Smitt M, Yu R, Leung AC, Wildiers H, collaborators TRs (2014) Trastuzumab emtansine versus treatment of physician's choice for pretreated HER2-positive advanced breast cancer (TH3RESA): a randomised, open-label, phase 3 trial. Lancet Oncol 15:689-699. https://doi.org/10.1016/S1470-2045(14)70178-0

8. Kaufman PA, Awada A, Twelves C, Yelle L, Perez EA, Velikova G, Olivo MS, He Y, Dutcus CE, Cortes J (2015) Phase III openlabel randomized study of eribulin mesylate versus capecitabine in patients with locally advanced or metastatic breast cancer previously treated with an anthracycline and a taxane. J Clin Oncol 33:594-601. https://doi.org/10.1200/JCO.2013.52.4892

9. Sobhani N, D’Angelo A, Pittacolo M, Roviello G, Miccoli A, Corona SP, Bernocchi O, Generali D, Otto T (2019) Updates on the CDK4/6 inhibitory strategy and combinations in breast cancer. Cells 8:321. https://doi.org/10.3390/cells8040321

10. Cristofanilli M, Turner NC, Bondarenko I, Ro J, Im SA, Masuda N, Colleoni M, DeMichele A, Loi S, Verma S, Iwata H, Harbeck N, Zhang K, Theall KP, Jiang Y, Bartlett CH, Koehler M, Slamon D (2016) Fulvestrant plus palbociclib versus fulvestrant plus placebo for treatment of hormone-receptor-positive, HER2-negative metastatic breast cancer that progressed on previous endocrine therapy (PALOMA-3): final analysis of the multicentre, double-blind, phase 3 randomised controlled trial. Lancet Oncol 17:425-439. https://doi.org/10.1016/S1470 -2045(15)00613-0

11. Sledge GW Jr, Toi M, Neven P, Sohn J, Inoue K, Pivot X, Burdaeva O, Okera M, Masuda N, Kaufman PA, Koh H, Grischke EM, Frenzel M, Lin Y, Barriga S, Smith IC, Bourayou N, Llombart-Cussac A (2017) MONARCH 2: abemaciclib in combination with fulvestrant in women with HR+/HER2- advanced breast cancer who had progressed while receiving endocrine therapy. J Clin Oncol 35:2875-2884. https://doi.org/10.1200/ JCO.2017.73.7585

12. Sledge GW Jr, Toi M, Neven P, Sohn J, Inoue K, Pivot X, Burdaeva O, Okera M, Masuda N, Kaufman PA, Koh H, Grischke EM, Conte P, Lu Y, Barriga S, Hurt K, Frenzel M, Johnston S, Llombart-Cussac A (2019) The effect of abemaciclib plus fulvestrant on overall survival in hormone receptor-positive, ERBB2negative breast cancer that progressed on endocrine therapyMONARCH 2: a randomized clinical trial. JAMA Oncol. https ://doi.org/10.1001/jamaoncol.2019.4782

13. Slamon DJ, Neven P, Chia S, Fasching PA, De Laurentiis M, Im S, Petrakova K, Bianchi GV, Esteva FJ, Martin M, Nusch A, Sonke GS, De la Cruz-Merino L, Beck JT, Pivot X, Sondhi M, Wang Y, Chakravartty A, Rodriguez-Lorenc K, Jerusalem G (2019) Overall survival (OS) results of the phase III MONALEESA-3 trial of postmenopausal patients (pts) with hormone receptor-positive (HR1), human epidermal growth factor 2-negative (HER22) advanced breast cancer (ABC) treated with fulvestrant (FUL) 6 ribociclib (RIB). Ann Oncol 30(suppl_5):v851-v934. https://doi. org/10.1093/annonc/mdz394

14. Torres-Guzman R, Calsina B, Hermoso A, Baquero C, Alvarez B, Amat J, McNulty AM, Gong X, Boehnke K, Du J, de Dios A, Beckmann RP, Buchanan S, Lallena MJ (2017) Preclinical characterization of abemaciclib in hormone receptor positive breast cancer. Oncotarget 8:69493-69507. https://doi.org/10.18632/ oncotarget. 17778

15. Dickler MN, Tolaney SM, Rugo HS, Cortes J, Dieras V, Patt D, Wildiers H, Hudis CA, O'Shaughnessy J, Zamora E, Yardley DA, Frenzel M, Koustenis A, Baselga J (2017) MONARCH 1, a phase II study of abemaciclib, a CDK4 and CDK6 inhibitor, as a 
single agent, in patients with refractory HR(+)/HER2(-) metastatic breast cancer. Clin Cancer Res 23:5218-5224. https://doi. org/10.1158/1078-0432.CCR-17-0754

16. Framework for FDA's real-world evidence program (2018). https ://www.fda.gov/media/120060/download. Accessed 31 July 2019

17. Sutter S (2019) Pink sheet - external control arms: better than single-arm studies but no replacement for randomization. https:// pink.pharmaintelligence.informa.com/PS124362/External-Contr ol-Arms-Better-Than-SingleArm-Studies-But-No-Replacemen t-For-Randomization. Accessed 31 July 2019

18. Booth CM, Karim S, Mackillop WJ (2019) Real-world data: towards achieving the achievable in cancer care. Nat Rev Clin Oncol 16:312-325. https://doi.org/10.1038/s41571-019-0167-7

19. Dreyer NA (2018) Advancing a framework for regulatory use of real-world evidence: when real is reliable. Ther Innov Regul Sci 52:362-368. https://doi.org/10.1177/2168479018763591

20. Curtis MD, Griffith SD, Tucker M, Taylor MD, Capra WB, Carrigan G, Holzman B, Torres AZ, You P, Arnieri B, Abernethy AP (2018) Development and validation of a high-quality composite real-world mortality endpoint. Health Serv Res 53:4460-4476. https://doi.org/10.1111/1475-6773.12872

21. Rubin DB (1980) Bias reduction using mahalanobis-metric matching. Biometrics 36:293-298

22. Hainmueller J (2012) Entropy balancing for causal effects: a multivariate reweighting method to produce balanced samples in observational studies. Polit Anal 20:25-46. https://doi.org/10.1093/pan/ mpr025

23. Corrigan-Curay J, Sacks L, Woodcock J (2018) Real-world evidence and real-world data for evaluating drug safety and effectiveness. JAMA 320:867-868. https://doi.org/10.1001/ jama.2018.10136

24. Cave A, Kurz X, Arlett P (2019) Real-world data for regulatory decision making: challenges and possible solutions for Europe. Clin Pharmacol Ther 106:36-39. https://doi.org/10.1002/cpt.1426
25. Blincyto® FDA review document (2014). https://www.accessdata .fda.gov/drugsatfda_docs/nda/2014/125557Orig 1s000MedRe dt.pdf

26. Blincyto® European Product Assessment Report (EPAR) (2015). http://www.ema.europa.eu/docs/en_GB/document_library/EPAR_ Public_assessment_report/human/003731/WC500198227.pdf

27. Bavencio® FDA review document (2016). https://www.accessdata .fda.gov/drugsatfda_docs/nda/2017/761049orig1s000multidisci pliner.pdf

28. Bavencio® European Product Assessment Report (EPAR) (2017). http://www.ema.europa.eu/docs/en_GB/document_library/EPAR_ Public_assessment_report/human/004338/WC500236649.pdf

29. Berger ML, Curtis MD, Smith G, Harnett J, Abernethy AP (2016) Opportunities and challenges in leveraging electronic health record data in oncology. Future Oncol 12:1261-1274. https://doi. org/10.2217/fon-2015-0043

30. Khozin S, Abernethy AP, Nussbaum NC, Zhi J, Curtis MD, Tucker M, Lee SE, Light DE, Gossai A, Sorg RA, Torres AZ, Patel P, Blumenthal GM, Pazdur R (2018) Characteristics of real-world metastatic non-small cell lung cancer patients treated with nivolumab and pembrolizumab during the year following approval. Oncologist 23:328-336. https://doi.org/10.1634/theon cologist.2017-0353

Publisher's Note Springer Nature remains neutral with regard to jurisdictional claims in published maps and institutional affiliations. 